\title{
Determinants of health-related quality of life in Iranian patients after recovery from COVID-19: Demographic influences and insomnia
}

\author{
Mohsen Saffari ${ }^{1,2}$, Hormoz Sanaeinasab ${ }^{1,2}$, Hojat Rashidi-Jahan ${ }^{1,2}$, Amir Pakpour Hajiagha ${ }^{3,4}$, Hosein \\ Mahmoudi $^{5}$, Faten Al-Zaben ${ }^{6}$, Harold George Koenig ${ }^{7,8,9}$ \\ ${ }^{1}$ Health Research Center, Life Style Institute, Baqiyatallah University of Medical Sciences, Tehran, Iran \\ ${ }^{2}$ Health Education Department, Faculty of Health, Baqiyatallah University of Medical Sciences, Tehran, Iran \\ ${ }^{3}$ Social Determinants of Health Research Center, Research Institute for Prevention of Non-Communicable Diseases, Qazvin University \\ of Medical Sciences, Qazvin, Iran \\ ${ }^{4}$ Department of Nursing, School of Health and Welfare, Jönköping University, Jönköping, Sweden \\ ${ }^{5}$ Trauma Research Center and Faculty of nursing, Baqiyatallah University of Medical Sciences, Tehran, Iran \\ ${ }^{6}$ Division of Psychiatry, Department of Medicine, King Abdulaziz University, Jeddah, Saudi Arabia \\ ${ }^{7}$ Department of Psychiatry and Behavioral Sciences, Duke University Medical Center, Durham, NC, USA \\ ${ }^{8}$ Department of Medicine, King Abdulaziz University, Jeddah, Saudi Arabia \\ ${ }^{9}$ School of Public Health, Ningxia Medical University, Yinchuan, China
}

\begin{tabular}{l}
\hline Article Info \\
\hline Article history: \\
Received May 30, 2021 \\
Revised Nov 8, 2021 \\
Accepted Nov 22, 2021 \\
\hline
\end{tabular}

Keywords:

COVID-19

Insomnia

Novel coronavirus

Quality of life

Recovery

\begin{abstract}
The current study sought to identify factors that may affect health-related quality of life (HRQoL) in patients recovering from COVID-19 infection in Iran. In a cross-sectional study 258 patients diagnosed with COVID-19, participants completed a questionnaire approximately one month after hospital discharge when demographic and clinical factors (including insomnia) and HRQoL were assessed. A logistic regression was used. Age, gender, marital status, education, having child, early physician visit, early diagnosis, early hospitalization, symptom type, Rhesus factor, and level of insomnia were associated with various components of HRQoL ( $\mathrm{p}<0.05)$. In multivariate analyses, poorer physical HRQoL was independently associated with female gender $(\mathrm{OR}=4.53 ; 95 \% \mathrm{CI}=2.22-2.29)$, initial symptom of cough $(\mathrm{OR}=2.73 ; 95 \% \mathrm{CI}=1.26-5.94)$, and insomnia $(\mathrm{OR}=2.74 ; 95 \%$ $\mathrm{CI}=1.22-6.14)$. Poorer mental HRQoL was associated with being age 40 years or older $(\mathrm{OR}=1.90 ; 95 \% \mathrm{CI}=1.02-3.54)$, female gender $(\mathrm{OR}=2.48$; $95 \% \mathrm{CI}=1.26-4.88)$, initial symptom being cough $(\mathrm{OR}=3.12$; 95\% $\mathrm{CI}=1.46-$ 6.68), and insomnia (sub-threshold insomnia, $\mathrm{OR}=3.19$; 95\% CI, 1.51-6.74, to severe insomnia, $\mathrm{OR}=3.86 ; 95 \% \mathrm{CI}=1.35-11.07)$. Healthcare professionals should be aware that older people, female gender, those with initial symptom of cough, and insomnia may be at greater risk for poor quality of life following hospital discharge.
\end{abstract}

This is an open access article under the CC BY-SA license.

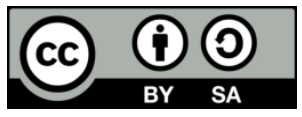

\section{Corresponding Author:}

Mohsen Saffari

Health Research Center, Life Style Institute, Baqiyatallah University of Medical Sciences

Sheykh-bahaei Street, Nosrati Alley, Tehran, Iran

Emails: Saffari.CHES@gmail.com, m.saffari@bmsu.ac.ir

\section{INTRODUCTION}

After diagnosis of the first case of the severe acute respiratory syndrome coronavirus 2 (SARSCoV-2) in China in early December 2019 and deadly respiratory disease accompanying it coronavirus disease 2019 (COVID-19), this has resulted in a worldwide pandemic involving more than 200 million persons and more than 4.2 million deaths as of August 10, 2021[1]. Both developed and underdeveloped regions of the 
world have suffered from the disease, although the United States is recognized as the country with the most cases and deaths, followed by India and Brazil [2]. Among Middle Eastern and South Asian countries, Iran is ranked third in terms of number of cases (4.7 million) and deaths (over 100,000 deaths). [1], [3]. Although several vaccines have now been developed against the development and spread of this novel coronavirus, the developments of new variants of the virus have raised questions about the long-term efficacy of such measures and how the disease may affect different aspects of life both among infected individuals and at-risk populations [4], [5].

COVID-19 has many physical, psychological, and social consequences at the individual and community level [6]. Fear and anxiety related to the development of the disease has caused persons to socially isolate themselves and reduced regular physical activity [7]. Those who have become infected by the virus usually have multiple neurological and physical complications such as muscle pain and fatigue that often persist for months after discharge from hospital [8]. In addition, during recovery period, COVID-19 patients often experience negative feelings as a result of these physical health consequences and being socially discriminated against, particularly anxiety, depression, and loneliness [9]. Other consequences of the disease include food security, reduction in family income, and unemployment, complicating the situation further and resulting in uncertainty about the future [10]. All of these factors may negatively affect the quality of life at the individual patient level and at the population level more generally.

Several studies have found that health related quality of life (HRQoL) is decreased among those recovered from the disease, including a reduction in well-being across physical, emotional, and social domains, for example $\mathrm{Qu}$ et al. [11] found that even three months after discharge more than half of people reported physical symptoms and nearly all of HRQoL domains scored lower than normal population. This finding in a same way found where Chen and colleagues assessed the patients for one month after discharge using SF-36 questionnaire [11]-[13]. There is a limited body of evidence that indicates demographic factors may affect HRQoL in those with COVID-19 [13], [14]. Unfortunately, however, the individual physical and mental components of HRQoL in patients recovering from the disease remain poorly understood. Assessing the HRQoL in these patients will improve our understanding of the disease process and complications that may threaten the well-being and recovery of patients in ways that healthcare professionals and decision makers may address [11]. Moreover, focusing on HRQoL as a comprehensive health outcome may help to determine patients' perceptions on different dimensions of life during recovery from COVID-19 and contribute to better estimate the burden of this disease [15], [16].

Assessment of HRQoL in patients with COVID-19 who are discharged from hospital and during the convalescence period is important because they may experience delayed complications that may not be present with other infections and include neurological, cardiovascular, and digestive complications [17]-[19]. Also, the possibility of recurrent symptoms such as cough, fatigue and dyspnea during recovery may distinctly affect HRQoL in such patients [20]. Continued respiratory system problems may decrease ability to exercise thereby negatively impacting emotional and physical recovery [21]. The impact of COVID-19 on activities of daily living several months after hospital discharge, as well as the general health of those who have recovered and their ability to return to individual and social roles in the community, remains unclear.

Sleep as a physiological process contributes to physical hemostasis and may directly or indirectly affect metabolic and immune processes [22]. Consequently, inability to obtain adequate sleep may significantly impact the recovery of physical and mental functioning in COVID-19 patients [23]. Insomnia and other sleep problems may trigger adaptive immune reactions and increased systemic inflammation [24]. Long-term sleep deprivation may also adversely affect natural killer cell activity [25]. Thus, poor quality of sleep and any change in circadian rhythm may weaken the body's resistance to and recovery form COVID19s. Other factors such physical disability, social isolation, and psychological reactions such as anxiety and fear of death may contribute to sleep problems [23]. A recent study reported that impaired sleep quality is present in nearly 34\% of patients with COVID-19 [26]. Sleep disorders in longtime may also predispose people to mental illnesses that such conditions also may interfere in the normal self-care behaviors resulting a higher susceptibility of infection with pathogens including Coronavirus [27].

After conducting a literature review on HRQoL in COVID-19 patients, the current study's authors decided to examine the quality of life of patients who had recovered from COVID-19 one month after hospital discharge. The purpose of this study was to examine demographic and clinical factors (e.g., time of diagnosis and sleep disturbance) related to HRQoL in these patients.

\section{RESEARCH METHOD}

\subsection{Design and participants}

Between August and November 2020, patients who had recovered from COVID-19 one month after their discharge from the hospital were surveyed in this cross-sectional study. This was a convenience sample 
of all COVID-19 patients at a general hospital referral center located in Tehran, Iran. Tehran as the capital of Iran registered the most cases of COVID-19 in Iran and it is the most crowded city in the country. The sample size was computed based on the Pedhazur estimation that there must be at least 30 observations per independent variable [28]. With 7-9 independent variables that may be included in the model, we calculated that at least 300 patients would need to be approached, anticipating a $10 \%$ attrition rate. A trained nurse conducted the first interview with all patients who were to be discharged during next few days based on their physician's decision. If they agreed to participate the contact information was obtained for an anticipated follow-up contact in one month post-discharge. When contacting participants one month later to complete the assessment, only 258 patients agreed to complete the survey. Inclusion criteria were: i) a confirmed diagnosis of novel coronavirus infection by polymerase chain reaction test (PCR), ii) hospital stay of at least for three days, iii) age 25 to 65 years old, and iv) able to speak and read Persian. Excluded were patients who experienced $\mathrm{s}$ recurrence of COVID-19, were forced to be re-hospitalized due to complications of the disease, were admitted to the intensive care unit (ICU) during hospitalization, had a history of serious psychological disorders such as psychosis, dementia or major depression, received treatment for insomnia before they developed COVID-19, and those who anticipated they would not be available 30-35 days after discharge. Patients who agreed to participate were contacted by telephone approximately one month after discharge, and were administered the interview. The purpose of the study and rights of participants to withdraw from the study at any stage were described prior to beginning of data collection. All participants provided written informed consent when registration and if any persons didn't have willingness to participate we replace them with other volunteer participants using convenient approach of sampling. The ethical review board of Baqiyatallah University of Medical Sciences approved the study protocol (ID: IR.BMSU.REC.1399.187).

\subsection{Measures}

Demographic and clinical information collected were age, gender, marital status, education, employment, smoking, blood group, Rhesus factor, and chronic comorbid medical conditions, and specific information about COVID-19 such as the interval between appearance of symptoms and first physician visit, time before symptom onset and diagnosis, the time between symptom onset and hospitalization, and the primary symptom that was manifested prior to diagnosis.

\subsubsection{Short form health survey (SF-8)}

The HRQoL was assessed using SF-8, which is an abbreviated version of the SF-36 questionnaire. The SF-8 consists of 8 items that make up eight subscales: general health (GH), physical functioning (PF), role limitation because of physical health problems (RP), role limitation because of emotional problems, (RE), bodily pain (BP), vitality (VT), social functioning (SF), and mental health (MH). These subscales make up two summary scores that are described as the physical component summary (PCS) and the mental component summary (MCS). The standardized score of the scale ranges from 0 to 100 with a mean value of 50 and standard deviation of 10 . Higher scores indicate better quality of life and the subscale scores as well as summary scores may be calculated individually (see data analysis below) [29]. The SF-12 and SF-36 have both been shown to have adequate psychometric properties in Iranian populations [30].

\subsubsection{Insomnia severity index (ISI)}

This scale was developed by Morin to assess subjective perceptions of sleep disturbance, and has been used to screen for insomnia in epidemiological studies. The Insomnia severity index (ISI) is a brief scale that includes seven items, which assess both nighttime and daytime symptoms, consequences, and concerns related to insomnia. All items are scored on a Likert type scale with five options (0-4) that indicate the severity of sleep problems during the past two weeks. The ISI total score ranges from 0 to 28 with higher scores indicating more severe insomnia. The following cut-off points have been recommended for interpretation of total scale scores: 0$7=$ normal or without clinically significant insomnia; 8-14=sub-threshold insomnia; $15-2=$ insomnia of moderate severity; and 22-28=severe, clinically significant insomnia. The Iranian version of the ISI has been shown to have acceptable reliability and validity in Persian speaking populations [31].

\subsection{Data analysis}

The mean (M) and standard deviation (SD) were used to describe continues variables, and categorical variables were presented as number $(\mathrm{N})$ and percent $(\%)$. The normality of item and scale responses were examined using the Shapiro-Wilk test. When the significance level of this test is greater than 0.05 , the distribution is considered normal. To evaluate associations between independent variables and HRQoL, bivariate analyses used the Student's t test and one way analysis of variance (ANOVA) followed by Tukey's post-hoc test. The subscale scores on the SF-8 as well as its summary scores were considered the 
dependent variables. The binary logistic regression was used to identify independent correlates of HRQoL. For this analysis, independent variables correlated with the dependent variables at an alpha level of less than 0.20 in bivariate analyses were included in the model. Summary scores (PCS and MCS) were entered as dependent variables. These dependent variables were mean-dichotomized so that those with scores lower than mean were given a score of 1 , while those with greater score than the mean were given score of 0 . Thus, when the resulting odds ratio (OR) is higher than 1, this indicates poorer HRQoL. Significance level was set at an alpha of less than 0.05 and all tests were performed using SPSS software for Windows version 24.0 (SPSS Inc, USA).

\section{RESULTS}

The mean age of the participants was 52.2 (15.0), and approximately $70 \%$ were 40 years or older. Approximately two-third of the participants were male and $80 \%$ were married. Only $37 \%$ of respondents had a university education and half of participants were unemployed. Approximately $10 \%$ of participants were smokers and more than half reported a chronic comorbid health condition such as hypertension, diabetes, or heart disease. Slightly over one-third of participants (36\%) reported they had contact with a person suspected of COVID-19 over past two weeks prior to hospital admission, and one-third reported they had been in a crowd such as a conference or familial get together during this period. The average of interval between the beginning of symptoms and seeking medical care was 4.5 ( $\mathrm{SD}=3.8$ ) days. Nearly $70 \%$ of participants had a confirmed diagnose of COVID-19 within three days after the visit. The most frequent symptom leading to presentation was fever (45\%). Blood groups $\mathrm{A}$ and $\mathrm{O}$ were present more frequently than others (Table 1) (see Appendix).

The bivariate associations between independent variables and subscales of HRQoL are presented in Table 2 (see in Appendix). There were significant associations present between SF-8 subscales and age, marital status, education, having a child, interval between physician visit and diagnosis, interval between initial symptoms and hospitalization, initial symptom presentation, and rhesus factor $(\mathrm{p}<0.05)$. Associations were present between gender and all subscale scores and overall PCS and MCS total scores. There was also a significant association between the presenting symptom of cough and both PCS and MCS total scores. All categories of insomnia were also significantly associated with PCS and MCS total scores.

Results of binary logistic regression are presented in Table 3 (see Appendix). The model was statistically significant $(\mathrm{X} 2=59.38 ; \mathrm{p}<0.001)$ and explained $21 \%$ to $28 \%$ of the variance in HRQLL based on Cox \& Snell and Nagelkerke R Square, respectively. Participants age 40 years or higher were nearly twice as likely to have poor mental HRQOL compared to those who were younger ( $\mathrm{OR}=1.90 ; 95 \% \mathrm{CI}=1.02-3.54)$. Women were over four times more likely to experience poorer physical HRQoL compared to men $(\mathrm{OR}=4.53$; 95\% CI=2.22-9.21) and were also over twice as likely to have low mental HRQoL compared to men $(\mathrm{OR}=2.48 ; 95 \% \mathrm{CI}=1.26-4.88)$. Those who had cough as their presenting symptom of COVID-19 were approximately three times more likely to have a low mental HRQoL than those with other presenting symptoms $(\mathrm{OR}=3.12 ; 95 \% \mathrm{CI}=1.46-6.68)$, as well as worse physical HRQoL (OR=2.73; 95\% $\mathrm{CI}=1.26-5.94)$. With regard to insomnia severity, participants who reported a moderate degree of insomnia were nearly three times more likely than others to report poor physical HRQoL (OR=2.74; 95\% $\mathrm{CI}=1.22-6.14)$, and those with severe insomnia were nearly four times as likely to have poor mental HRQoL $(\mathrm{OR}=3.86$; 95\% $\mathrm{CI}=1.35-11.07)$.

\section{DISCUSSION}

The purpose of the present study was to examine demographic and clinical factors that may be influential in determining health-related quality of life in those who have been recently recovered from COVID-19. Findings revealed that several characteristics were associated with a higher likelihood of poor physical and mental quality of life at one month after hospital discharge. Being age 40 years or old, female, having cough as a presenting symptom, and having insomnia may increase have poor physical or mental HRQoL at this time.

Although research in this area is limited, a few studies have examined factors associated with quality of life in COVID-19 patients. For example, Arab-Zozani et al. [14] assessed HRQoL using EQ-5D questionnaire in 420 patients 2-3 weeks after hospital discharge in a study that took place in the Yazd province of Iran. Consistent with the findings in the present study, these investigators found that female gender and older age were significant predictors of HRQoL in such patients. However, other factors such as education, employment status, having diabetes or heart failure, and being admitted to the intensive care unit was also associated with HRQoL in that study, in contrast to the findings here. However, there were several differences between that study and the present study that may help to explain the difference in findings. First, assessment of HRQoL was assessed using different measures, with perhaps one being a more sensitive measure of quality of life than the other. Second, we excluded patients who had a history of ICU admission during hospitalization. Therefore, those with more serious cases of COVID-19 were excluded from the current sample. Third, here patients were assessed one month after hospital discharge rather than 2-3 weeks 
after discharge, and therefore a longer follow-up period may lead to improvements in outcome for patients with certain demographic and clinical characteristics. The factor that distinguished the present study from the Arab-Zozani et al. [14] study is the examination of insomnia as a correlate of HRQoL, which to our knowledge is the first time this has been examined in Iran.

In another study, Chen et al. [13] examined correlates of HRQoL using the SF-36 in patients recovered from COVID-19 at one-month follow up in the country of China. These investigators assessed 361 patients, including patients with severe cases of COVID-19 with an average age of $47 \pm 13$ years. The gender distribution of the sample indicated an approximately equal frequency of men and women. They found significant associations between several subscales of the SF-36 (e.g., PF, RE, GH, and VT) and age, gender, having a chronic illness, length of hospital stay, and smoking history, the only factor associated with mental health QoL (MCS) was gender. Women were over twice as likely $(\mathrm{OR}=2.2)$ to have a poor MCS as men. This finding was similar to that in the present study. However, with regard to physical HLQoL, we found that women were more than four times likely to have poor life quality in this area compared to men. This finding indicates that the physical component of HRQoL may be been more impaired than the mental health component in women. Other reasons for differences in the findings between the present and the Chen et al. [13] study may have been different settings, different cultural environments, and different demographic characteristics.

Although to our knowledge no studies have yet examine the effects of sleep quality and HRQoL in Iran, several studies that assessed these among COVID-19 patients in other countries [11], [23], [32], [33]. However, almost all of these studies examine this association in currently hospitalized patients, not among COVID-19 patients after hospital discharge. Therefore, as noted earlier, the present study is the first to examine sleep problems in patients during the convalescence period following discharge. In a cross-sectional study, Akinci and Basar [23] examined the association between sleep quality and psychological status in 189 patients with COVID-19. They found that factors such as timing the hospital and depressive symptoms correlated with poorer sleep quality. This finding is similar to that in the present study which found that problems with sleep may be a risk factor for reductions in mental health QoL during the recovery period from COVID-19. In another study, Jiang et al. [32] examined psychological distress and sleep quality among COVID-19 patients in Wuhan, China, finding a significant relationship between the two. In addition, these investigators also found that age, gender, and severity of disease symptoms were significantly associated with mental health problems such as anxiety and depression in these patients. These findings are also similar to those from the present study, confirming that demographic factors may play an important role in the HRQoL of COVID-19 patients during their recovery period. Furthermore, insomnia itself is associated with physical health problems that can negatively affect QoL in patients with COVID-19 [34] and may also adversely affect immune functioning, which may interfere with recovery from infection or resistance to it [35]. Another negative effect of a low sleep quality in such patients is related to cognitive ability of these patients. Studies have shown people with sleep problems over time may lose their cognitive functions and therefore couldn't perform their job activities or social roles normally that may affect their social health and may be resulted to a reduction in their HRQoL score [36].

The current study also found that when COVID-19 presents initially with the symptom of cough (rather than shortness of breath or other symptoms was associated with poor physical and mental components of HRQoL). An explanation for this finding may be that patients with coughing often have more severe respiratory symptoms. Respiratory involvement may have poorer outcomes from the disease and a more persistent course of symptoms, adversely affecting HRQoL during recovery. COVID-19 patients with more extensive respiratory infection are known to have longer hospitalizations than those with non-respiratory involvement and poorer outcomes more generally [37].

\subsection{Study limitations}

Several limitations of the current study limit the generalization and interpretation of the findings. First, participants were a convenience sample of volunteers from a single healthcare center, limiting generalizability to all Iranian patients hospitalized and discharged from other regions of the country. However, no particular cultural or geographical factors are likely all that different in Tehran than other areas of the country, except being an urban setting. Second, there was refusal rate of $14 \%$, which was greater than the $10 \%$ estimated. However, an $86 \%$ response rate is relatively high for patients in recovery from an illness as serious as COVID-19 with many symptoms even during recovery that may interfere with a patient's desire to participate in a study. Furthermore, the sample size was adequate for the statistical analyses that were performed. Third, there are many other factors such as socio-economic status, intensive care hospitalization, family medical history, race/ethnic factors, and other demographic and clinical characteristics that may influence quality of life that were not assessed in the current study. Fourth, the participants only were followed for one month after discharge whereas a longer follow up may contribute us to find which risk factors may affect participants over time. However due to our limited resources to follow patients at longer periods we 
limited it to just one month. Finally, this was a cross-sectional study that makes it impossible to determine whether the characteristics identified here were causal in their effects on HRQoL or vice-versa. Only prospective studies can provide information relevant to causal inference, and these are a high priority for future research.

\section{CONCLUSION}

The present study found that older age, female gender, being married, having children in the home, having a presenting symptom of cough, having a longer interval between symptoms and hospitalization, and having insomnia are all associated with worse HRQoL in patients recovering from COVID-19 one month after hospital discharge. Healthcare professionals and planners need to be aware that these characteristics may identify COVID-19 patients who are at greater risk of developing reductions in health-related quality of life following discharge from the hospital, and therefore consider developing programs to support such patients. This will help to minimize the negative impact that COVID-19 has on patients, their families, and the communities in which they live.

\section{ACKNOWLEDGEMENTS}

The authors would like to express their thanks to clinical research development unit of Baqiyatallah Hospital that provided technical support to conduct this study.

\section{REFERENCES}

[1] World Health Organization, "Weekly epidemiological update on COVID-19 - 10 August 2021," World Health Organization, 2021. https://www.who.int/publications/m/item/weekly-epidemiological-update-on-covid-19--10-august-2021 (accessed Feb. 09, 2021).

[2] L. P. Breitling, "Global epidemiology and socio-economic development correlates of the reproductive ratio of COVID-19," International Health, pp. 1-6, Mar. 2021, doi: 10.1093/inthealth/ihab006.

[3] World Health Organization, "WHO coronavirus (COVID-19) dashboard.” https://covid19.who.int/table (accessed May 25, 2021).

[4] S. P. Teo, "Review of COVID-19 vaccines and their evidence in older adults," Annals of Geriatric Medicine and Research, vol. 25, no. 1, pp. 4-9, Mar. 2021, doi: 10.4235/agmr.21.0011.

[5] S. H. Hodgson, K. Mansatta, G. Mallett, V. Harris, K. R. W. Emary, and A. J. Pollard, "What defines an efficacious COVID-19 vaccine? A review of the challenges assessing the clinical efficacy of vaccines against SARS-CoV-2," The Lancet Infectious Diseases, vol. 21, no. 2, pp. e26--e35, Feb. 2021, doi: 10.1016/S1473-3099(20)30773-8

[6] V. J. Clemente-Suárez, A. A. Dalamitros, A. I. Beltran-Velasco, J. Mielgo-Ayuso, and J. F. Tornero-Aguilera, "Social and psychophysiological consequences of the COVID-19 pandemic: An extensive literature review," Frontiers in Psychology, vol. 11, Dec. 2020, doi: 10.3389/fpsyg.2020.580225.

[7] K. Tsamakis et al., "COVID-19 and its consequences on mental health (Review)," Experimental and Therapeutic Medicine, vol. 21, no. 3, p. 244, Jan. 2021, doi: 10.3892/etm.2021.9675.

[8] S. Soltani et al., "COVID-19 associated central nervous system manifestations, mental and neurological symptoms: a systematic review and meta-analysis," Reviews in the Neurosciences, vol. 32, no. 3, pp. 351-361, Apr. 2021, doi: 10.1515/revneuro-2020-0108.

[9] F. Jia and X. Liu, "Using social media to analyze public psychological status during the recovery period of COVID-19 in China," Journal of public health (Oxford, England), vol. 43, no. 2, pp. e238--e240, Jun. 2021, doi: 10.1093/pubmed/fdaa152.

[10] G. Bonaccorsi et al., "Economic and social consequences of human mobility restrictions under COVID-19," in Proceedings of the National Academy of Sciences of the United States of America, Jul. 2020, vol. 117, no. 27, pp. 15530-15535, doi: 10.1073/pnas.2007658117.

[11] G. Qu et al., "Health-related quality of life of COVID-19 patients after discharge: A multicenter follow-up study," Journal of Clinical Nursing, vol. 30, no. 11-12, pp. 1742-1750, Jun. 2021, doi: 10.1111/jocn.15733.

[12] P. Santus et al., "Changes in quality of life and dyspnoea after hospitalization in COVID-19 patients discharged at home," Multidisciplinary Respiratory Medicine, vol. 15, no. 1, Oct. 2020, doi: 10.4081/mrm.2020.713.

[13] K.-Y. Chen, T. Li, F.-H. Gong, J.-S. Zhang, and X.-K. Li, "Predictors of health-related quality of life and influencing factors for COVID-19 patients, a follow-up at one month," Frontiers in Psychiatry, vol. 11, Jul. 2020, doi: 10.3389/fpsyt.2020.00668.

[14] M. Arab-Zozani, F. Hashemi, H. Safari, M. Yousefi, and H. Ameri, "Health-related quality of life and its associated factors in COVID-19 patients," Osong Public Health and Research Perspectives, vol. 11, no. 5, pp. 296-302, Oct. 2020, doi: 10.24171/j.phrp.2020.11.5.05

[15] V. H. Rice, Ed., Handbook of Stress, Coping, and Health Implications for Nursing Research, Theory, and Practice, 2nd ed. California: SAGE Publications, 2012.

[16] K. H. Park, A. R. Kim, M. A. Yang, S. J. Lim, and J. H. Park, "Impact of the COVID-19 pandemic on the lifestyle, mental health, and quality of life of adults in South Korea," PLOS ONE, vol. 16, no. 2, p. e0247970, Feb. 2021, doi: 10.1371/journal.pone.0247970.

[17] A. Iqubal, M. K. Iqubal, F. Hoda, A. K. Najmi, and S. E. Haque, "COVID-19 and cardiovascular complications: an update from the underlying mechanism to consequences and possible clinical intervention," Expert Review of Anti-Infective Therapy, vol. 19, no. 9, pp. 1083-1092, Sep. 2021, doi: 10.1080/14787210.2021.1893692.

[18] A. Jarrahi et al., "Neurological consequences of COVID-19: What have we learned and where do we go from here?," Journal of Neuroinflammation, vol. 17, no. 1, p. 286, Dec. 2020, doi: 10.1186/s12974-020-01957-4.

[19] Z. Y. Dong, B. J. Xiang, M. Jiang, M. J. Sun, and C. Dai, “The prevalence of gastrointestinal symptoms, abnormal liver function, digestive system disease and liver disease in COVID-19 infection: a systematic review and meta-analysis," Journal of Clinical Gastroenterology, vol. 55, no. 1, pp. 67-76, Jan. 2021, doi: 10.1097/MCG.0000000000001424.

[20] C. F. V. Takeda et al., "Case report: recurrent clinical symptoms of COVID-19 in healthcare professionals: a series of cases from Brazil," American Journal of Tropical Medicine and Hygiene, vol. 103, no. 5, pp. 1993-1996, Nov. 2020, doi: 10.4269/ajtmh.200893. 
[21] A. Méndez-Giménez, J. A. Cecchini, J. Fernández-Río, and A. Carriedo, "Physical activity and prevention of depressive symptoms in the Spanish population during confinement due to COVID-19," Psicothema, vol. 33, no. 1, pp. 111-117, 2021, doi: 10.7334/psicothema2020.294

[22] E. Maurovich-Horvat, T. Z. Pollmächer, and K. Sonka, "The effects of sleep and sleep deprivation on metabolic, endocrine and immune parameters," Prague Medical Report, vol. 109, no. 4, pp. 275-285, 2008.

[23] T. Akıncı and H. Melek Başar, "Relationship between sleep quality and the psychological status of patients hospitalised with COVID-19," Sleep Medicine, vol. 80, pp. 167-170, Apr. 2021, doi: 10.1016/j.sleep.2021.01.034

[24] T. Lange, S. Dimitrov, and J. Born, "Effects of sleep and circadian rhythm on the human immune system: Annals of the New York Academy of Sciences," Annals of the New York Academy of Sciences, vol. 1193, no. 1, pp. 48-59, Apr. 2010, doi: 10.1111/j.1749-6632.2009.05300.x.

[25] M. E. P. Sousa et al., "Invariant Natural Killer T cells resilience to paradoxical sleep deprivation-associated stress," Brain, Behavior, and Immunity, vol. 90, pp. 208-215, Nov. 2020, doi: 10.1016/j.bbi.2020.08.018.

[26] J. Deng et al., "The prevalence of depression, anxiety, and sleep disturbances in COVID-19 patients: a meta-analysis," Annals of the New York Academy of Sciences, vol. 1486, no. 1, pp. 90-111, Feb. 2021, doi: 10.1111/nyas.14506.

[27] N. Vindegaard and M. E. Benros, "COVID-19 pandemic and mental health consequences: Systematic review of the current evidence,” Brain, Behavior, and Immunity, vol. 89, pp. 531-542, Oct. 2020, doi: 10.1016/j.bbi.2020.05.048.

[28] E. J. Pedhazur, Multiple Regression in Behavioral Research : Explanation and Prediction, 3rd ed. California: Harcourt Brace or Harcourt Press, 1997.

[29] S. Ghafari, F. Ahmadi, M. Nabavi, K. Anoshirvan, R. Memarian, and M. Rafatbakhsh, "Effectiveness of applying progressive muscle relaxation technique on quality of life of patients with multiple sclerosis," Journal of Clinical Nursing, vol. 18, no. 15, pp. 2171-2179, Aug. 2009, doi: 10.1111/j.1365-2702.2009.02787.x.

[30] A. Montazeri, M. Vahdaninia, S. J. Mousavi, and S. Omidvari, "The Iranian version of 12-item short form health survey (SF-12): Factor stucture, internal consistency and construct validity," BMC Public Health, vol. 9, no. 1, p. 341, Dec. 2009, doi: 10.1186/1471-2458-9-341.

[31] K. Sadeghniiat-Haghighi, A. Montazeri, A. Khajeh-Mehrizi, S. Nedjat, and O. Aminian, "The insomnia severity index: crosscultural adaptation and psychometric evaluation of a Persian version," Quality of Life Research, vol. 23, no. 2, pp. 533-537, Mar. 2014, doi: 10.1007/s11136-013-0489-3.

[32] Z. Y. Jiang et al., "Psychological distress and sleep quality of COVID-19 patients in Wuhan, a lockdown city as the epicenter of COVID-19," Journal of Psychiatric Research, vol. 136, pp. 595-602, Apr. 2021, doi: 10.1016/j.jpsychires.2020.10.034.

[33] Y. F. Ma et al., "Prevalence of depression and its association with quality of life in clinically stable patients with COVID-19," Journal of Affective Disorders, vol. 275, pp. 145-148, Oct. 2020, doi: 10.1016/j.jad.2020.06.033.

[34] B. Sivertsen et al., "Insomnia as a risk factor for ill health: results from the large population-based prospective HUNT Study in Norway,” Journal of Sleep Research, vol. 23, no. 2, pp. 124-132, Apr. 2014, doi: 10.1111/jsr.12102.

[35] D. J. Taylor, K. Kelly, M. L. Kohut, and K. S. Song, "Is insomnia a risk factor for decreased influenza vaccine response?," Behavioral Sleep Medicine, vol. 15, no. 4, pp. 270-287, Jul. 2017, doi: 10.1080/15402002.2015.1126596.

[36] N. Goel, H. Rao, J. S. Durmer, and D. F. Dinges, "Neurocognitive consequences of sleep deprivation," Seminars in Neurology, vol. 29, no. 4, pp. 320-339, Sep. 2009, doi: 10.1055/s-0029-1237117.

[37] A. Hernández-Biette, J. Sanz-Santos, L. Boix-Palop, A. N. Rolón, M. Martínez-Palau, and A. de la Sierra Iserte, "Risk factors for later hospitalization of patients discharged from an emergency department with nonsevere COVID-19 symptoms," Emergencias, vol. 32 , no. 6 , pp. 413-415, 2020.

\section{BIOGRAPHIES OF AUTHORS}

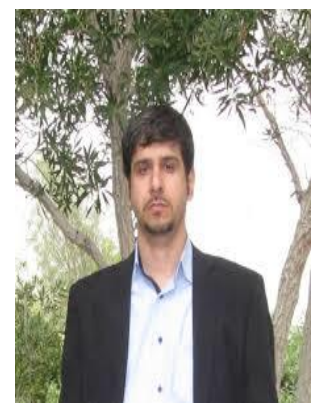

Mohsen Saffari (D) BI SC P is a health education and promotion specialist. He works as a professor in the Baqiyatallah University of Medical sciences and likes research on topics such as health related quality of life, health literacy, spirituality and social determinants of the health. He has published more than one hundred articles in English and Persian regarding health issues. Also more than 10 books regarding health education and promotion in Persian have been published by him. He can be contacted at email: saffari.ches@gmail.com.

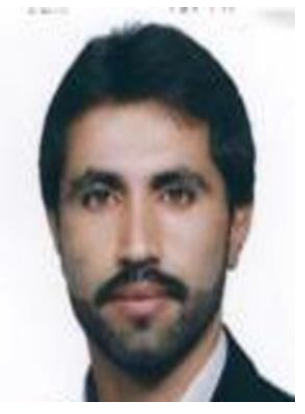

Hormoz Sanaeinasab (iD) $\mathrm{SC}$ S $\mathrm{P}$ is a Professor of Health education and promotion in the Baqiyatallah University of Medical Sciences. His research interests are health education interventions in people especially with chronic disorders. He is one the pioneers to establish and extend the health related activities in the Iranian council of health education and promotion. He is currently the editor-in-chief in the journal of military health promotion. He can be contacted at email: sanaei1343@gmail.com. 

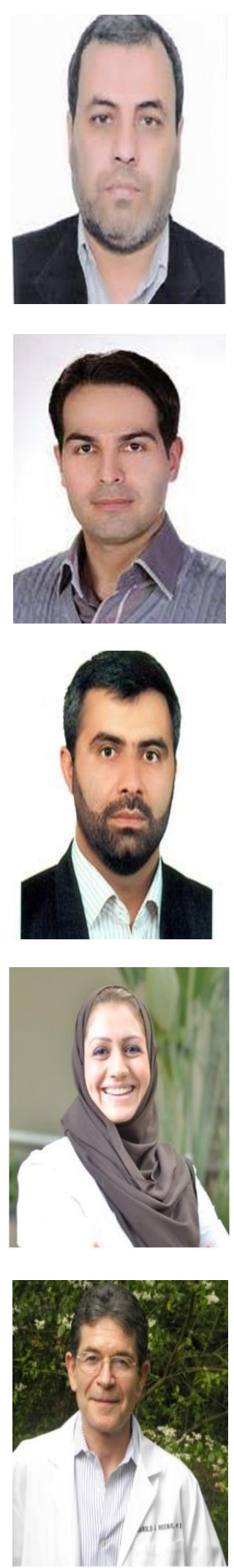

Hojat Rashidi-Jahan (D) S SC P is Assistant professor of health education in the Baqiyatallah University of medical sciences. He interested in research topics on health education intervention and disease prevention especially for chronic diseases. He teaches educational technology, disease control and prevention, school health and worksite health promotion for public health students. He can be contacted at email: hojatr73@gmail.com.

Amir Pakpour Hajiagha (D) SI SC P is Full professor of Health education. He was recognized as the Top Iranian researcher in Health sciences in Razi festival. Some other his important achievements are: distinguished Researcher Award in Qazvin Province, Top Young Investigator Award: Islamic Educational, Scientific, and Cultural Organization (ISESCO), Top One Percent on ESI researchers in 2021. He works in the Qazvin University of Medical Sciences and is the affiliated researcher, School of Health and Welfare, Jönköping University, Sweden. He can be contacted at email: pakpour_amir@yahoo.com.

Hosein Mahmoudi (iD I8 SC P is a Professor of Nursing Education in the Baqiyatallah University of Medical Sciences. He is also affiliated at the Trauma Research Center, Baqiyatallah University of Medical Sciences. He is interested to do studies in Nursing Sciences. Also he likes qualitative and quantitative studies on patients admitted in emergence care units. He can be contacted at email: h.mahmoudi@bmsu.ac.ir.

Faten Al-Zaben (D) IS SC P has received her Bachelor degree in Medicine and Surgery (MBBCH) in 2000 in Saudi Arabia. She Joined the Residency Program in psychiatry 2003, University of Western Ontario (London, Canada) and obtained the Canadian Board of psychiatry (FRCPc) in 2008. She has completed a fellowship program in Mood and Anxiety disorders May 2010, and joined king Abdulaziz university hospital, Faculty of Medicine, Psychiatry Department 2011, and is currently the head of Psychiatry unit. She is a Fellow of the royal college of physicians and surgeons of Canada; Member of the Canadian psychiatric association, the Ontario psychiatric association, the American psychiatric Association, and the abuse protection committee at King Abdulaziz University Hospital. She is interested to work on patients with autism. She can be contacted at email: faten_zaben@yahoo.com.

Harold George Koenig (D) $\mathrm{SC}$ S $\mathrm{P}$ is a Professor of Psychiatry and Behavioral Sciences and Associate Professor of Medicine in Duke University Medical Center. He also works as the Senior Fellow in the Center for the Study of Aging and Human Development. His primary research is focused on studying the effects of religion and spirituality on health. Dr. Koenig has over 550 scientific peer-reviewed academic publications, nearly 100 book chapters, and 55 books. His research has been featured on many national and international TV programs and hundreds of national and international radio programs and newspapers/magazines. He is the recipient of the 2012 Oskar Pfister Award from the American Psychiatric Association and the 2013 Gary Collins award from the American Association of Christian Counselors. He is the former editor-in-chief of the International Journal of Psychiatry in Medicine, and is the currently an Associate Editor of the Journal of Religion and Health. He can be contacted at email: harold.koenig@duke.edu. 


\section{APPENDIX}

Table 1. Sample characteristics $(n=258)$

\begin{tabular}{|c|c|}
\hline Variables & Number $(\%)$ \\
\hline \multicolumn{2}{|l|}{ Age } \\
\hline$<40$ & $78(30.2)$ \\
\hline$\geq 40$ & $180(69.8)$ \\
\hline \multicolumn{2}{|l|}{ Gender } \\
\hline Male & $168(65.1)$ \\
\hline Female & $90(34.9)$ \\
\hline \multicolumn{2}{|l|}{ Marital status } \\
\hline Single (including widows and divorces) & $52(20.2)$ \\
\hline Married & $206(79.8)$ \\
\hline \multicolumn{2}{|l|}{ Education } \\
\hline High school or less & $162(62.8)$ \\
\hline University & $96(37.2)$ \\
\hline \multicolumn{2}{|l|}{ Employment } \\
\hline Employed & $127(49.2)$ \\
\hline Unemployed & $131(50.8)$ \\
\hline \multicolumn{2}{|l|}{ Having child } \\
\hline Yes & $211(81.8)$ \\
\hline No & $47(18.2)$ \\
\hline \multicolumn{2}{|l|}{ Smoking } \\
\hline Yes & $25(9.7)$ \\
\hline No & $211(81.8)$ \\
\hline Previously smoke & $22(8.5)$ \\
\hline \multicolumn{2}{|l|}{ Chronic comorbidity } \\
\hline Yes & $147(57.0)$ \\
\hline No & $111(43.0)$ \\
\hline \multicolumn{2}{|l|}{ Type of comorbidity } \\
\hline Diabetes & $40(15.5)$ \\
\hline Hypertension & $43(16.7)$ \\
\hline Heart diseases & $26(10.1)$ \\
\hline Renal diseases & $6(2.3)$ \\
\hline Pulmonary diseases & $17(6.6)$ \\
\hline Others & $15(5.8)$ \\
\hline \multicolumn{2}{|l|}{ COVID-19 specific data } \\
\hline Identified contact with a symptomatic individual at least two weeks before contraction (yes) & $93(36.0)$ \\
\hline Travel to an area with a high prevalence at least two weeks before contraction (yes) & 30 (11.6) \\
\hline $\begin{array}{l}\text { Presence in a crowd at least two weeks before contraction (conference, wedding ceremony) } \\
\text { (yes) }\end{array}$ & $79(30.6)$ \\
\hline Going to a healthcare center at least two weeks before contraction (hospital, clinic) (yes) & $95(36.8)$ \\
\hline \multicolumn{2}{|l|}{ Interval between symptoms and a physician visit (day) } \\
\hline$<5 \quad-120$ & $181(70.2)$ \\
\hline$\geq 5$ & $77(29.8)$ \\
\hline \multicolumn{2}{|l|}{ Interval between physician visit and diagnosis (day) } \\
\hline$<3 \quad-180$ & $176(68.2)$ \\
\hline$\geq 3$ & $82(31.8)$ \\
\hline \multicolumn{2}{|l|}{ Interval between symptoms and hospitalization (day) } \\
\hline$<5$ & $173(67.0)$ \\
\hline$\geq 5$ & $85(33.09)$ \\
\hline \multicolumn{2}{|l|}{ First leading symptom } \\
\hline Fever & $116(45.0)$ \\
\hline Cough & $63(24.4)$ \\
\hline Fatigue & $14(5.4)$ \\
\hline Dyspnea & $34(13.2)$ \\
\hline Nausea/vomiting & $9(3.5)$ \\
\hline Sore throat & $8(3.1)$ \\
\hline Others & $14(5.4)$ \\
\hline \multicolumn{2}{|l|}{ Blood group } \\
\hline A & $74(28.7)$ \\
\hline B & $42(16.3)$ \\
\hline $\mathrm{O}$ & $68(26.4)$ \\
\hline $\mathrm{AB}$ & $19(7.4)$ \\
\hline Unknown & $55(21.3)$ \\
\hline \multicolumn{2}{|l|}{ Rhesus factor (Rh) } \\
\hline+ & $175(67.8)$ \\
\hline- & $28(10.9)$ \\
\hline Unknown & $55(21.3)$ \\
\hline
\end{tabular}

Int J Public Health Sci, Vol. 11, No. 1, March 2022: 220-231 
Table 2. Relationship between health-related quality of life, demographic, and clinical characteristics

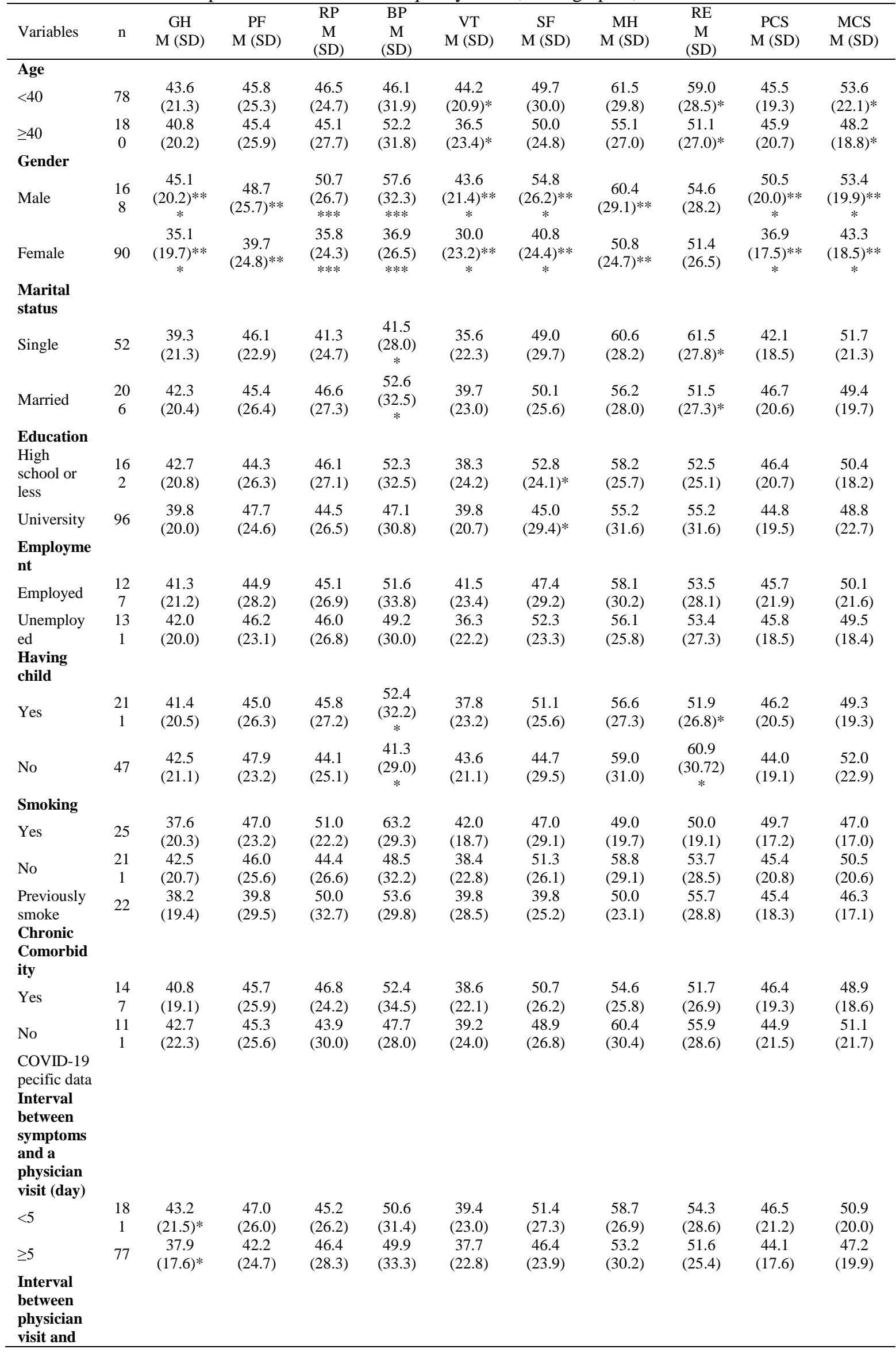




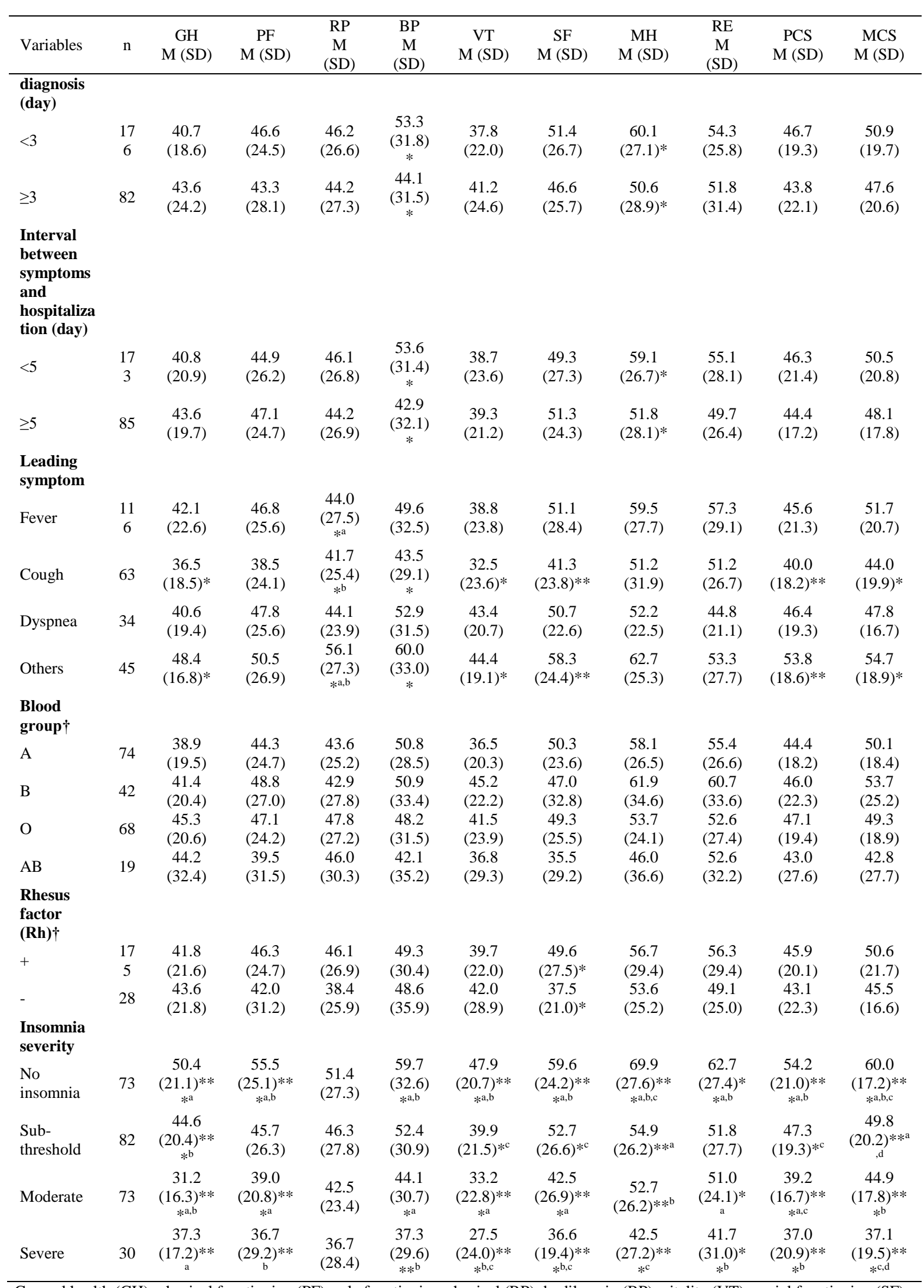

General health (GH); physical functioning (PF); role functioning-physical (RP); bodily pain (BP), vitality (VT); social functioning (SF); mental health $(\mathrm{MH})$; role functioning-emotional (RE); physical component summary (PCS); mental component summary (MCS) $\dagger$ Comparisons are based on known cases $(\mathrm{n}=203) ; * \mathrm{p}$ value $<0.05 ; * * \mathrm{p}$ value $<0.01 ; * * * \mathrm{p}$ value $<0.001$

In ANOVA, values with same letters are different significantly. 
Table 3. Logistic regression analysis examining the association between demographics, clinical characteristics, and health-related quality of life assessed by the SF-8

\begin{tabular}{|c|c|c|c|c|c|}
\hline \multirow[t]{2}{*}{ Independent variables } & \multirow[t]{2}{*}{ Categories } & \multicolumn{2}{|l|}{ PCS } & \multicolumn{2}{|l|}{ MCS } \\
\hline & & OR $(95 \% \mathrm{CI})$ & $\mathrm{p}$ value & OR $(95 \% \mathrm{CI})$ & $\mathrm{p}$ value \\
\hline \multirow[t]{2}{*}{ Age } & $<40$ & Ref & - & Ref & - \\
\hline & $\geq 40$ & $0.77(0.40-1.48)$ & 0.440 & $1.90(1.02-3.54)$ & 0.043 \\
\hline \multirow[t]{2}{*}{ Gender } & Male & Ref & - & Ref & - \\
\hline & Female & $4.53(2.22-9.21)$ & $<0.001$ & $2.48(1.26-4.88)$ & 0.008 \\
\hline \multirow[t]{2}{*}{ Marital status } & Married & Ref & - & Ref & - \\
\hline & Single & $1.54(0.67-3.51)$ & 0.302 & $0.53(0.24-1.15)$ & 0.109 \\
\hline \multirow[t]{2}{*}{ Symptom start and visit } & $<5$ & Ref & - & Ref & - \\
\hline & $\geq 5$ & $1.35(0.69-2.63)$ & 0.378 & $1.40(0.73-2.68)$ & 0.307 \\
\hline \multirow[t]{2}{*}{ Symptom start and diagnosis } & $<3$ & Ref & - & Ref & - \\
\hline & $\geq 3$ & $1.28(0.67-2.43)$ & 0.455 & $0.61(0.32-1.14)$ & 0.121 \\
\hline \multirow[t]{4}{*}{ Leading symptom } & Fever & Ref & - & Ref & - \\
\hline & Cough & $2.73(1.26-5.94)$ & 0.011 & $3.12(1.46-6.68)$ & 0.003 \\
\hline & Dyspnea & $2.15(0.89-5.19)$ & 0.087 & $1.96(0.79-4.86)$ & 0.143 \\
\hline & Others & $0.75(0.33-1.71)$ & 0.496 & $1.48(0.67-3.27)$ & 0.332 \\
\hline \multirow[t]{5}{*}{ Blood group } & B & Ref & - & Ref & - \\
\hline & $\mathrm{A}$ & $1.19(0.50-2.80)$ & 0.695 & $0.92(0.39-2.17)$ & 0.848 \\
\hline & $\mathrm{O}$ & $0.85(0.36-2.00)$ & 0.710 & $1.18(0.49-2.81)$ & 0.710 \\
\hline & $\mathrm{AB}$ & $2.62(0.68-10.06)$ & 0.158 & $2.72(0.71-10.44)$ & 0.144 \\
\hline & Unknown & $0.76(0.29-2.01)$ & 0.582 & $0.44(0.17-1.14)$ & 0.092 \\
\hline \multirow[t]{2}{*}{ Rhesus factor (Rh) } & Positive & Ref & - & Ref & - \\
\hline & Negative & $0.90(0.34-2.36)$ & 0.826 & $2.13(0.77-5.94)$ & 0.146 \\
\hline \multirow[t]{4}{*}{ Insomnia severity } & No insomnia & Ref & - & Ref & - \\
\hline & Sub-threshold & $1.09(0.52-2.30)$ & 0.815 & $3.19(1.51-6.74)$ & 0.002 \\
\hline & Moderate & $2.74(1.22-6.14)$ & 0.015 & $4.22(1.89-9.39)$ & $<0.001$ \\
\hline & Severe & $2.41(0.86-6.74)$ & 0.094 & $3.86(1.35-11.07)$ & 0.012 \\
\hline
\end{tabular}

Physical component summary (PCS); mental component summary (MCS); confidence Interval (CI); odds ratio (OR); reference category (Ref) 